\title{
Hepatic Gene Expression Associated With Macrophage and Oxidative Stress of Simple Steatosis and Non-Alcoholic Steatohepatitis Model Rats Using DNA Microarray Analysis
}

\author{
Keiichiro Ohba ${ }^{1,3 *}$, Toshio Kumai ${ }^{3}$, Shinichi Iwai ${ }^{1}$, Minoru Watanabe ${ }^{4}$, Go Koizumi ${ }^{1,3}$,Masayuki Arai ${ }^{1}$, Kanji Furuya ${ }^{1}$, Go Oda ${ }^{3}$, Naoki Matsumoto ${ }^{2}$, \\ Shinichi Kobayashi ${ }^{1}$ and Katsuji Oguchi ${ }^{1}$ \\ ${ }^{1}$ Department of Pharmacology, Showa University School of Medicine, 1-5-8 Hatanodai, Shinagawa-ku, Tokyo 142-8555, Japan \\ ${ }^{2}$ Department of Pharmacology, St. Marianna University School of Medicine, 2-16-1 Sugao, Miyamae-ku, Kawasaki, Kanagawa 216-8511, Japan \\ ${ }^{3}$ Department of Pharmacogenomics, St. Marianna University Graduate School of Medicine, 2-16-1 Sugao, Miyamae-ku, Kawasaki, Kanagawa 216-8511, Japan \\ ${ }^{4}$ Institute for Animal Experimentation, St. Marianna University Graduate School of Medicine, 2-16-1 Sugao, Miyamae-ku, Kawasaki, Kanagawa 216-8511, Japan
}

\begin{abstract}
Aim: To clarify the mechanism governing progression of Non-Alcoholic Steatohepatitis (NASH), we examined hepatic gene expression associated with macrophage and oxidative stress/inflammation, which plays an important role in the progression of Non-Alcoholic Fatty Liver Disease (NAFLD) in simple steatosis (SS) model and NASH model rats.
\end{abstract}

Methods: Four-month-old male Spontaneously Hypertensive Hyperlipidemic Rats (SHHR) and Sprague-Dawley (SD) rats were each divided into two groups: SD rats received a high-fat diet and $30 \%$ sucrose solution (HFDS) as SS model rats and SHHR received the HFDS as NASH model rats. Microarray analysis was performed on the liver of these rats at eight months of age to select those gene sets, e.g., "genes correlated with progression of NAFLD" and "genes expressed exclusively in NASH", which are related to macrophage or oxidative stress/inflammation.

Results: Thirteen genes were selected from the microarray analysis data. Four genes were associated with macrophage: acid phosphatase 5, tartrate-resistant (Acp5), a member of the RAS oncogene family (Rab8a), scavenger receptor class B, member 2 (Scarb2) and CD36 molecule (Cd36). Nine genes were associated with oxidative stress/inflammation: translocator protein (Tspo), prostaglandin I2 synthase (Ptgis), tumor necrosis factor receptor superfamily, member 9 (Tnfrsf9), glutathione S-transferase alpha 5 (Gsta5), regucalcin (Rgn), glutathione S-transferase kappa 1 (Gstk1), disabled homolog 2, mitogen-responsive phosphoprotein (Dab2), glutathione S-transferase mu 5 (Gstm5) and flavin-containing monooxygenase 5 (Fmo5). Acp5, Tspo, Ptgis, Tnfrsf, Gsta5 (upregulated) and Rab8a, Rgn, Gstk1 (down-regulated) were included in genes correlated with progression of NAFLD. Scarb2, Cd36, Dab2 Gstm5 (up-regulated) and Fmo5 (down-regulated) were included in genes expressed in only $\mathrm{NASH}$ model rats.

Conclusion: We hypothesized that scavenger receptor class B and glutathione S-transferase play an important role in the progression from simple NAFLD to NASH. Our results afford beneficial data regarding therapeutic targets of progression of NAFLD/NASH.

Keywords: Spontaneously hypertensive hyperlipidemic rats (SHHR) (SHHR); Non-alcoholic steatohepatitis (NASH); Non-alcoholic fatty liver disease (NAFLD); Microarray

\section{Introduction}

Non-alcoholic Fatty Liver Disease (NAFLD) is defined as the accumulation of lipid, primarily triacylglycerols, in individuals who do not consume large quantities of alcohol. NAFLD encompasses a spectrum of disease ranging from Simple Steatosis (SS) to inflammatory steatohepatitis with increasing levels of fibrosis and ultimately cirrhosis [1]. NAFLD, which is strongly correlated with obesity and insulin resistance, is recognized as representing the hepatic manifestation of the metabolic syndrome [2]. Non-Alcoholic Steatohepatitis (NASH) is a progressive form of NAFLD that is diagnosed on the basis of histopathological features [3]. The prevalence of the metabolic syndrome, which is rapidly increasing due to hyperalimentation and a sedentary lifestyle [4], is reflected by the increasing prevalence of NAFLD and associated complications [5]. NAFLD can be quite unobtrusive; in contrast, NASH is a serious condition, with nearly one quarter of affected patients developing cirrhosis, which, in turn, increases the risk of subsequent progression to hepatocellular carcinoma [6]. Despite recent advances in the elucidation of the complex metabolic and inflammatory pathways involved in NAFLD, the pathogenesis of steatosis and progression to steatohepatitis and fibrosis/cirrhosis remains incompletely understood $[2,7]$.
Few rat models lend themselves to the evaluation of atherosclerosis; as a result, Spontaneously Hypertensive Hyperlipidemic Rats (SHHRs) were developed as a stable model of early vascular degeneration [8], SHHRs display persistently high systolic blood pressure (above 150 $\mathrm{mmHg}$ ) and plasma total cholesterol levels exceeding $150 \mathrm{mg} / \mathrm{dL}$. In addition, vascular intimal lesions and lipid deposits have been observed under endothelial cells in the aorta of SHHRs, but not in spontaneously hyperlipidemic rats or controls [9]. Moreover, invasive changes occur in the subendothelium of SHHR when nitric oxide production is inactivated, followed by a high fat diet and sucrose water treatment (HFDS) [10]; moreover, a previous study noted visceral fat accumulation and increased oxidative stress in SHHR-HFDS $[11,12]$

Corresponding author: Keiichiro Ohba, Department of Pharmacology, Showa University School of Medicine, 1-5-8, Hatanodai, Shinagawa-ku, Tokyo 142-8555 Japan, Tel: +81 33784 8125; Fax: +81 33787 4790; E-mail: kei@oba.name

Received October 01, 2012; Accepted November 15, 2012; Published Novembe 17,2012

Citation: Ohba K, Kumai T, Iwai S, Watanabe M, Koizumi G, et al. (2012) Hepatic Gene Expression Associated With Macrophage and Oxidative Stress of Simple Steatosis and Non-Alcoholic Steatohepatitis Model Rats Using DNA Microarray Analysis. J Metabolic Synd 1:115. doi:10.4172/2167-0943.1000115

Copyright: ( 2012 Ohba K, et al. This is an open-access article distributed unde the terms of the Creative Commons Attribution License, which permits unrestricted use, distribution, and reproduction in any medium, provided the original author and source are credited. 
Citation: Ohba K, Kumai T, Iwai S, Watanabe M, Koizumi G, et al. (2012) Hepatic Gene Expression Associated With Macrophage and Oxidative Stress of Simple Steatosis and Non-Alcoholic Steatohepatitis Model Rats Using DNA Microarray Analysis. J Metabolic Synd 1:115. doi:10.4172/2167-0943.1000115

Page 2 of 7

We detected hepatocyte ballooning and steatosis with HFDS feeding for 9-13 months. Severe fibrosis and cell inflammation around the central vein were observed in SHHR-HFDS but were only slightly detectable in SD-HFDS [13]; thus, SD-HFDS and SHHR-HFDS were employed as rat models of simple steatosis and NASH, respectively.

Numerous researchers have applied microarray analysis in order to clarify the underlying mechanism governing NAFLD; however, these investigations noted only the genes correlated with lipid metabolism. The metabolic abnormalities and gene expressions of liver in terms of the pathological progression of early stage NASH remain unclear.

Microarray analysis was performed in the current study to clarify the pathogenetic mechanisms governing early stage NASH and to evaluate SD-HFDS and SHHR-HFDS as models of simple steatosis and $\mathrm{NASH}$, respectively.

\section{Materials and Methods}

\section{Animals and samples}

Four-month-old male SHHRs and SD rats were divided into two groups each: a control group, which received a regular diet (ND; CE2; CLEA Japan Inc., Tokyo, Japan), and a HFDS-fed group. The regular diet consisted of $8.9 \%$ water, $25.4 \%$ protein, $4.4 \%$ fat, $4.1 \%$ fiber, $6.9 \%$ carbohydrate and $50.3 \%$ nitrogen-free extracts (caloric value: $342.2 \mathrm{kcal} / 100 \mathrm{~g}$ ). The high-fat diet (HFD) consisted of $8.2 \%$ water, $23.4 \%$ protein, $11.0 \%$ fat, $3.8 \%$ fiber, $6.3 \%$ carbohydrate and $46.3 \%$ nitrogen-free extracts (caloric value: $378.0 \mathrm{kcal} / 100 \mathrm{~g}$ ). The regular diet was available to all groups ad libitum until the age of four months; subsequently, the two HFDS groups received the high-fat diet with $30 \%$ sucrose solution ad libitum for four months. Thus, rats of 8 months of age were obtained. This study utilized SHHRs characterized by systolic blood pressure over $150 \mathrm{mmHg}$, as determined by the tailcuff method (PS-100; Riken Kaihatsu, Tokyo, Japan). The rats were housed in a semi-barrier system under controlled room temperature $\left(23 \pm 1^{\circ} \mathrm{C}\right)$, humidity $(55 \pm 5 \%)$ and lighting (lights on from $6 \mathrm{AM}$ to $6 \mathrm{PM}$ ). All experiments were conducted according to the "Guiding Principles for the Care and Use of Laboratory Animals" of Showa University [12].

\section{Statistical analysis}

Data were analyzed employing the Mann-Whitney U-test. Correlations were calculated with the Pearson's product moment correlation coefficient. All data are expressed as mean \pm S.E.M. $\mathrm{p}<0.05$ was considered significant.

\section{Preparation and biochemical determination of plasma samples}

Blood samples, which were obtained from the inferior vena cava under pentobarbital anesthesia $(35 \mathrm{mg} / \mathrm{kg}$, intraperitoneal administration), were mixed with $3.2 \%$ sodium citrate solution in a volume ratio of 9:1. After 15 minutes of centrifugation at 3,000 rpm, the supernatant, as citrated plasma, was analyzed. Rats were sacrificed by decapitation, after which the liver and visceral fat (VisF) were isolated and weighed under the pentobarbital anesthesia.

Plasma levels of total cholesterol and total triglycerides were determined employing commercially available kits (Cholesterol E-test and Triglyceride E-test, respectively; Wako Pure Chemical Industries Ltd., Tokyo, Japan). Plasma levels of Aspartate Aminotransferase (AST) and Alanine Aminotransferase (ALT) were determined with the Transaminase CII-test (Wako) [11]. Oxidative stress was measured in rat plasma utilizing the d-ROMs test (Free Radical Elective Evaluator; Wismerll Co. Ltd., Tokyo, Japan) by gently mixing $20 \mu$ l of plasma sample and $1 \mathrm{~mL}$ of buffer solution in a cuvette, prior to the addition of $10 \mu \mathrm{L}$ of the chromogenic substrate. After mixing, the cuvette was incubated immediately in the thermostatic block of the analyzer for 5 minutes at $37^{\circ} \mathrm{C}$; subsequently, the absorbance at $505 \mathrm{~nm}$ was recorded. Measurements are expressed as Carr units, with 1 Carr corresponding to $0.8 \mathrm{mg} / \mathrm{L} \mathrm{H}_{2} \mathrm{O}_{2}$ [12].

\section{Morphological study}

The fresh left lobe of liver was harvested from all rats and stored in

\begin{tabular}{|c|c|c|c|c|c|c|c|}
\hline & $\mathbf{N}$ & $B W(g)$ & $L W(g)$ & Vis Fat(g) & LW/100gBW & Vis Fat $/ 100 \mathrm{gBW}$ & d-ROMS \\
\hline SD-ND & 8 & $574.1 \pm 8.5$ & $14.7 \pm 0.3$ & $17.3 \pm 0.3$ & $2.6 \pm 0.0$ & $3.0 \pm 0.1$ & $281.8 \pm 8.7$ \\
\hline SD-HFDS & 8 & $590 \pm 12.1$ & $38.2 \pm 1.4$ & $19.3 \pm 1.4$ & $6.5 \pm 0.1$ & $3.3 \pm 0.2$ & $482.0 \pm 35.0$ \\
\hline SHHR-ND & 6 & $565 \pm 7.7$ & $16.3 \pm 0.5$ & $14.7 \pm 1.1$ & $2.9 \pm 0.1$ & $2.6 \pm 0.2$ & $360.7 \pm 13.0$ \\
\hline SHHR-HFDS & 6 & $671.2 \pm 14.9$ & $48.3 \pm 0.5$ & $50.4 \pm 3.8$ & $7.2 \pm 0.1$ & $7.5 \pm 0.4$ & $616.8 \pm 23.4$ \\
\hline & & $* 3 * 5 * 6$ & $* 1 * 2 * 3 * 4 * 5 * 6$ & ${ }^{*} 2 * 3 * 4{ }^{*} 5^{\star} 6$ & ${ }^{*} 1 * 2 * 3 * 4 * 5 * 6$ & ${ }^{*} 2 * 3 * 4 * 5 * 6$ & ${ }^{*} 1 * 2 * 3 * 4 * 5 * 6$ \\
\hline
\end{tabular}

Body weight (BW), visceral fat (VisF), plasma glucose (Glu), hemoglobin A1c (HbA1c), total cholesterol (TC), total triglycerides (TG), aspartate aminotransferase (AST), alanine aminotransferase (ALT) and diacron reactive oxidative metabolites (d-ROMs) in Sprague-Dawley rats receiving normal diet (SD-ND) and SD-high-fat diet with sucrose (SD-HFDS) as well as in spontaneously hypertensive hyperlipidemic rats (SHHR-ND and SHHR-HFDS) at 8 months of age. The results are presented as mean \pm S.E.M.

Student $\mathrm{t}$ test unpaired $\mathrm{p}<0.05$ for *1, SD-ND vs. SD-HFDS ; ${ }^{*} 2, \mathrm{SD}-\mathrm{ND}$ vs. SHHR-ND ; *3, SD-ND vs. SHHR-HFDS ; *4, SD-HFDS vs. SHHR-ND ; 5 , SD-HFDS vs. SHHR-HFDS ; *6, SHHR-ND vs. SHHR-HFDS

Table 1a: Biochemical characteristics of SD-ND, SD-HFDS, SHHR-ND and SHHR-HFDS at 8 months of age.

\begin{tabular}{|c|c|c|c|c|c|c|c|}
\hline & $\mathbf{N}$ & Glu(mg/dl) & HbA1c & T-Cho(mg/dl) & $T G(m g / d l)$ & AST & ALT \\
\hline SD-ND & 8 & $150.9 \pm 7.9$ & $2.7 \pm 0.1$ & $65.8 \pm 4.9$ & $77.0 \pm 8.2$ & $141.9 \pm 15.3$ & $53.9 \pm 8.9$ \\
\hline SD-HFDS & 8 & $162.6 \pm 5.1$ & $2.8 \pm 0.1$ & $109.9 \pm 10.3$ & $72.8 \pm 5.4$ & $264.1 \pm 46.9$ & $71.1 \pm 10.0$ \\
\hline SHHR-ND & 6 & $135.8 \pm 8.9$ & $2.6 \pm 0.1$ & $140.2 \pm 9.9$ & $125.0 \pm 9.2$ & $140.3 \pm 9.2$ & $47.5 \pm 4.8$ \\
\hline \multirow[t]{2}{*}{ SHHR-HFDS } & 6 & $162.8 \pm 3.6$ & $2.9 \pm 0.1$ & $457.3 \pm 42.5$ & $84.7 \pm 6.6$ & $170.7 \pm 12.0$ & $62.2 \pm 18.0$ \\
\hline & & $* 4 * 6$ & *1*2 & $* 3 * 5 * 6$ & ${ }^{*} 2 * 4 * 6$ & $* 1 * 4$ & \\
\hline
\end{tabular}

Body weight (BW), visceral fat (VisF), plasma glucose (Glu), hemoglobin A1c (HbA1c), total cholesterol (TC), total triglycerides (TG), aspartate aminotransferase (AST), alanine aminotransferase (ALT) and diacron reactive oxidative metabolites (d-ROMs) in Sprague-Dawley rats receiving normal diet (SD-ND) and SD-high-fat diet with sucrose (SD-HFDS) as well as in spontaneously hypertensive hyperlipidemic rats (SHHR-ND and SHHR-HFDS) at 8 months of age. The results are presented as mean \pm S.E.M. Student $t$ test unpaired $\mathrm{p}<0.05$ for *1, SD-ND vs. SD-HFDS ; 2 , SD-ND vs. SHHR-ND ; *3, SD-ND vs. SHHR-HFDS ; 4 , SD-HFDS vs. SHHR-ND ; 5 , SD-HFDS vs. SHHR-HFDS ; *6, SHHR-ND vs. SHHR-HFDS 
Citation: Ohba K, Kumai T, Iwai S, Watanabe M, Koizumi G, et al. (2012) Hepatic Gene Expression Associated With Macrophage and Oxidative Stress of Simple Steatosis and Non-Alcoholic Steatohepatitis Model Rats Using DNA Microarray Analysis. J Metabolic Synd 1:115. doi:10.4172/2167-0943.1000115

saline on ice, after which it was dissected from the surrounding tissues and fixed in $10 \%$ neutral buffered formalin ( $\mathrm{pH} 7.4$; Wako). Sections of the liver were stained with Hematoxylin-Eosin (HE) and Masson Trichrome (MT).

\section{RNA extraction and microarray hybridization}

A sample of each liver specimen was stored at $-80^{\circ} \mathrm{C}$ until the microarray analysis.

Total RNA was purified using an RNeasy Kit (Qiagen, Germany) per the manufacturer's manual. Cy3-labeled cRNA was obtained from 200 ng total RNA with the Agilent Low Input Quick Amp Labeling Kit. Cy3labeled cRNA was hybridized to Whole Rat genome Oligo Microarray ver $3.0(4 \times 44 \mathrm{k})$ according to the manufacturer's hybridization protocol. After the washing step, microarray slides were analyzed with an Agilent Microarray scanner B version; the default settings were applied for all parameters. Microarray expression data were obtained utilizing Agilent Feature Extraction software ver 10.5.1; the default settings were applied for all parameters. The raw data and associated sample interpretation were loaded and processed by GeneSpring ver11 (Tomy Digital Biology). Four experiments were performed; data were expressed as mean values.

\section{Results}

\section{Comparison of the four groups}

Tables $1 \mathrm{a}$ and $1 \mathrm{~b}$ displays body weight and Liver Weight (LW) as well as the levels of VisF, plasma glucose, hemoglobin A1C, total cholesterol, total triglycerides, AST, ALT and d-ROMs in SD-ND, SD-HFDS, SHHR-ND and SHHR-HFDS. In both the SD and SHHR groups, VisF and LW were elevated significantly or tended to be elevated following ingestion of HFDS. VisF and LW in SHHR-ND and SHHRHFDS increased markedly in comparison with SD-ND and SD-HFDS, respectively. The level of oxidative stress (d-ROMs) demonstrated meaningful elevation after ingestion of HFDS. The level of d-ROMs in SHHR-ND and SHHR-HFDS increased significantly in comparison with SD-ND and SD-HFDS, respectively. Plasma hemoglobin A1C, AST and ALT levels were unchanged among the SD and SHHR groups.

\section{Morphological study}

Figures 1 and 2 presents the results of the morphological study

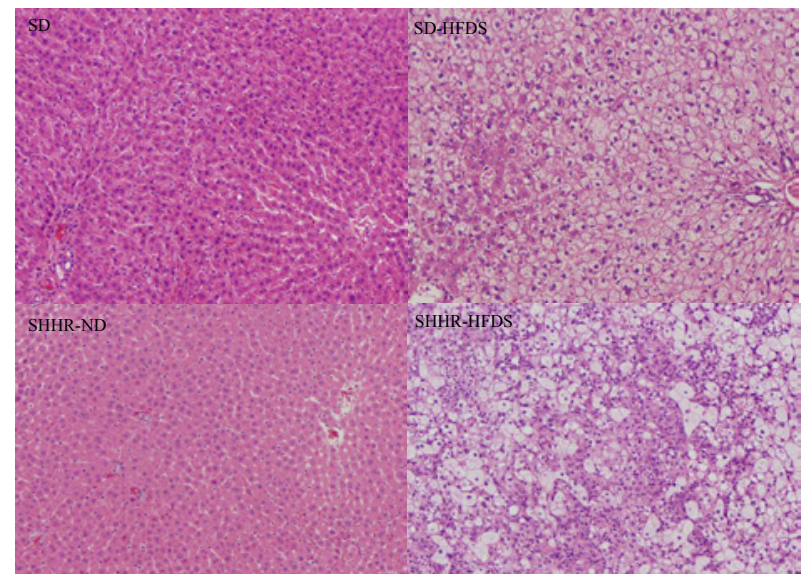

Figure 1: Effects of high fat diet and $30 \%$ sucrose on the liver of Spontaneously Hypertensive Hyperlipidemic Rats (SHHR) and SD rats (Hematoxylin -eosin stain) SD-ND; SD rats fed with normal diet (ND), SD-HFDS; SD rats fed with high fat diet and $30 \%$ sucrose (HFDS), SHHR-ND; SHHR fed with ND, SHHR-HFDS; SHHR fed with HFDS.

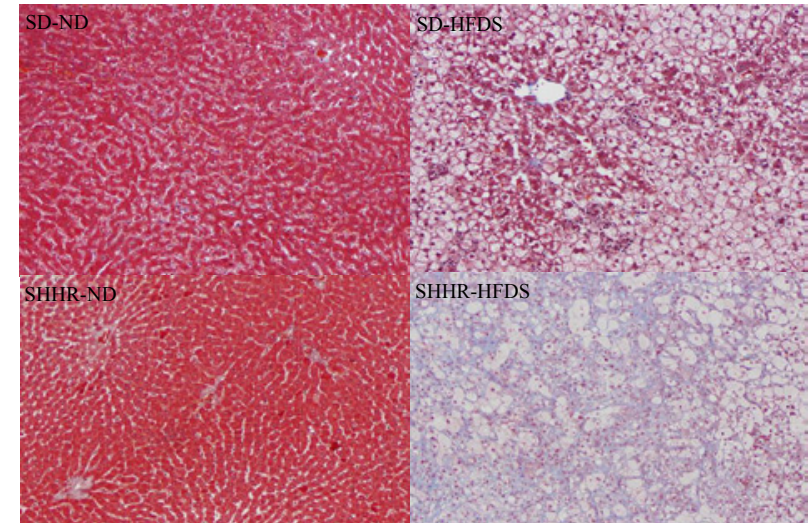

Figure 2: Effects of high fat diet and $30 \%$ sucrose on the liver of Spontaneously Hypertensive Hyperlipidemic Rats (SHHR) and SD rats (Masson trichrome stain) SD-ND; SD rats fed with Normal Diet (ND), SD-HFDS; SD rats fed with high fat diet and $30 \%$ sucrose (HFDS), SHHR-ND; SHHR fed with ND, SHHR-HFDS; SHHR fed with HFDS.

with a representative sample of liver from each group of SHHR and SD rats at eight months stained with HE and MT. Hepatocyte ballooning and steatosis are well recognized with HFDS feeding. Fibrosis and cell inflammation, which were observed in SHHR-HFDS, were slightly detectable in SD-HFDS.

\section{DNA microarray analysis}

On the basis of quality control, 8388 genes were detected. Genes were analyzed statistically employing Student $\mathrm{T}$ test unpaired (p-value computation; asymptotic, multiple testing correction; BenjaminiHochberg, p-value cut off; <0.05) to select pathophysiologically important genes: "Genes correlated with progression of NAFLD", which met all criteria; $\mathrm{p}<0.05$ for SD-ND vs. SD-HFDS, SHHR-ND vs. SHHR-HFDS, SD-ND vs. SHHR-ND, SD-HFDS vs. SHHR-HFDS. "Genes expressed exclusively in NASH", which met all criteria; $\mathrm{p}<0.05$ for SD-ND vs. SD-HFDS, $\mathrm{p}<0.05$ for SHHR-ND vs. SHHR-HFDS, SDND vs. SHHR-ND, SD-HFDS vs. SHHR-HFDS. Subsequently, those genes demonstrating identical regulation among all four criteria were obtained as up-regulated or down-regulated. Forty-four and 49 genes were correlated with up-regulation and down-regulation, respectively, in terms of NAFLD progression. Seventy-seven and 32 genes were correlated with up-regulation and down-regulation, respectively, in NASH. Finally, 13 genes were selected from the macrophage infiltrationand oxidative stress-related genes per microarray analysis data.

\section{Discussion}

Several earlier studies have established a relationship between obesity and lifestyle-related diseases. In particular, visceral obesity is directly correlated with the clustering of lifestyle-related diseases, leading to various ailments [14-16]. Our previous report demonstrated that visceral fat accumulation is closely associated with increased oxidative stress [12]. We disclosed that oxidative stress, matrix metalloproteinases (MMPs) and tissue inhibitors of metalloproteinases (TIMPs) play an important role in NASH progression in the liver of NASH model rats fed HFDS for 9-13 months. We also determined that hepatocyte ballooning and steatosis are well recognized with HFDS feeding for 9-13 months; moreover, severe fibrosis and cell inflammation were readily apparent around the central vein in SHHR-HFDS but only slightly detectable in SD-HFDS [13]. This study examined hepatic gene expression profiles in the early stage of NASH in the liver of NASH model rats receiving the HFDS for 8 months. 
Citation: Ohba K, Kumai T, Iwai S, Watanabe M, Koizumi G, et al. (2012) Hepatic Gene Expression Associated With Macrophage and Oxidative Stress of Simple Steatosis and Non-Alcoholic Steatohepatitis Model Rats Using DNA Microarray Analysis. J Metabolic Synd 1:115. doi:10.4172/2167-0943.1000115

Page 4 of 7

The most accepted theory regarding the explanation of progression from simple steatosis to NASH is the "two-hit hypothesis" wherein fat accumulation is sufficient to induce progression to steatohepatitis, rendering the liver more susceptible to a "second hit" that, once imposed upon the steatotic liver, causes further aberrations that culminate in the development of NASH $[17,18]$. A key factor in this "second hit" is oxidative stress [13]. Biochemical characteristics of SHHR-HFDS indicate that SHHR-HFDS at 8 months of age may develop metabolic syndrome leading to NAFLD.

A second feature of this study was the statistical method utilized to clarify the pathophysiological mechanism governing progression from simple steatosis to NASH. Based on our previous report, e.g., severe fibrosis and cell inflammation around the central vein were readily apparent in SHHR-HFDS but only slightly detectable in SD-HFDS [13], two groups of genes were extracted: "Genes correlated with progression of NAFLD" and "Genes expressed exclusively in NASH". The former category contained those genes changed in expression, correlated with liver steatosis, oxidative stress and visceral fat accumulation; in other words, "first-hit" induced the gene expressions and "second-hit" enhanced them. The latter category consisted of those genes induced not by "first-hit" but by "second-hit", associated with the aforementioned background. Recently, it was reported that macrophage infiltration and oxidative stress are an initiating event in NASH $[19,20]$. Therefore, 13 genes were selected from the candidate genes per microarray analysis data and classified as macrophage-related or oxidative stress/ inflammation-related genes (Tables $2 \mathrm{a}-2 \mathrm{c}$ ). Four genes were associated with macrophage: acid phosphatase 5, tartrate-resistant (Acp5), a member of the RAS oncogene family (Rab8a), scavenger receptor class B, member 2 (Scarb2) and CD36 molecule (Cd36). Furthermore, nine genes were associated with oxidative stress/inflammation: translocator protein (Tspo), prostaglandin I2 synthase (Ptgis), tumor necrosis factor receptor superfamily, member 9 (Tnfrsf9), glutathione S-transferase alpha 5 (Gsta5), regucalcin (Rgn), glutathione S-transferase kappa 1

\begin{tabular}{|c|c|c|c|c|}
\hline & \multicolumn{2}{|c|}{$\begin{array}{l}\text { Genes correlated with } \\
\text { progression of NAFLD }\end{array}$} & \multicolumn{2}{|c|}{$\begin{array}{l}\text { Genes expressed } \\
\text { exclusively in NASH }\end{array}$} \\
\hline & Up-regulated & Down-regulated & Up-regulated & Down-regulated \\
\hline macrophage & Acp5 & Rab8a & $\begin{array}{l}\text { Scarb2 } \\
\text { Cd36 }\end{array}$ & \\
\hline $\begin{array}{l}\text { oxidative stress } \\
\text { inflammation }\end{array}$ & $\begin{array}{l}\text { Tspo } \\
\text { Ptgis } \\
\text { Tnfrsf } \\
\text { Gsta5 }\end{array}$ & $\begin{array}{l}\text { Rgn } \\
\text { Gstk1 }\end{array}$ & $\begin{array}{l}\text { Dab2 } \\
\text { Gstm5 }\end{array}$ & Fmo5 \\
\hline
\end{tabular}

Table 2a: Macrophage- and oxidative stress/inflammation-related genes acid phosphatase 5, tartrate resistant (Acp5), translocator protein (Tspo), prostaglandin 12 synthase (Ptgis), tumor necrosis factor receptor superfamily, member 9 (Tnfrsf,), glutathione S-transferase alpha 5 (Gsta5), member RAS oncogene family (Rab8a), regucalcin (Rgn), glutathione S-transferase kappa 1 (Gstk1), scavenger receptor class B, member 2 (Scarb2), CD36 molecule (Cd36), disabled homolog 2, mitogenresponsive phosphoprotein (Dab2), glutathione S-transferase mu 5 (Gstm5), flavin-containing monooxygenase 5 (Fmo5)

\begin{tabular}{|c|c|c|c|c|}
\hline $\begin{array}{l}\text { Gene } \\
\text { Symbol }\end{array}$ & $\begin{array}{l}\text { SD-HFDS } \\
\text { vs. SD-ND }\end{array}$ & $\begin{array}{l}\text { SHHR-ND } \\
\text { vs. SD-ND }\end{array}$ & $\begin{array}{l}\text { SHHR-HFDS } \\
\text { vs. SD-HFDS }\end{array}$ & $\begin{array}{l}\text { SHHR-HFDS } \\
\text { vs. SHHR-ND }\end{array}$ \\
\hline \multicolumn{5}{|l|}{ Up-regulated genes } \\
\hline Acp5 & 2.21 & 2.38 & 3.26 & 3.02 \\
\hline Tspo & 2.29 & 2.17 & 2.11 & 2.22 \\
\hline Gsta5 & 4.00 & 6.34 & 3.44 & 1.84 \\
\hline Ptgis & 1.90 & 4.84 & 13.50 & 5.30 \\
\hline Tnfrsf9 & 6.51 & 7.75 & 4.25 & 3.57 \\
\hline \multicolumn{5}{|c|}{ Down-regulated genes } \\
\hline Rgn & -2.31 & -3.32 & -2.51 & -1.75 \\
\hline Rab8a & -1.31 & -1.31 & -1.22 & -1.22 \\
\hline Gstk1 & -1.97 & -1.64 & -1.68 & -2.02 \\
\hline
\end{tabular}

Table 2b: Changed genes correlated with steatosis HFDS treatment significantly increased or decreased gene expression in SD and SHHR. Meaningful differences were observed between comparison groups. Each mean fold value is presented.

\begin{tabular}{|l|l|l|l|l|}
\hline $\begin{array}{l}\text { Gene } \\
\text { Symbol }\end{array}$ & $\begin{array}{l}\text { SD-HFDS } \\
\text { vs. SD-ND }\end{array}$ & $\begin{array}{l}\text { SHHR-ND } \\
\text { vs. SD-ND }\end{array}$ & $\begin{array}{l}\text { SHHR-HFDS } \\
\text { vs. SD-HFDS }\end{array}$ & $\begin{array}{l}\text { SHHR-HFDS } \\
\text { vs. SHHR-ND }\end{array}$ \\
\hline Up-regulated genes & \multicolumn{5}{|l|}{} \\
\hline Scarb2 & 1.07 & 1.02 & 1.12 & 1.18 \\
\hline Dab2 & 1.58 & 2.14 & 3.05 & 2.25 \\
\hline Gstm5 & 1.22 & 1.79 & 3.36 & 1.54 \\
\hline Cd36 & 1.21 & 1.73 & 4.57 & 2.19 \\
\hline Down-regulated genes & \multicolumn{5}{|l|}{} \\
\hline Fmo5 & -1.52 & -2.17 & -3.09 & -2.17 \\
\hline
\end{tabular}

Table 2(c): Changed genes correlated with steatohepatitis HFDS treatment significantly increased or decreased gene expression in only SHHR. HFD treatment did not lead to meaningful differences between the SD HFDS and SD ND groups. Each mean fold value is presented.

(Gstk1), disabled homolog 2, mitogen-responsive phosphoprotein (Dab2), glutathione S-transferase mu 5 (Gstm5) and flavin-containing monooxygenase 5 (Fmo5). Acp5, Tspo, Ptgis, Tnfrsf, Gsta5 (upregulated) as well as Rab8a, Rgn and Gstk1 (down-regulated) were included in the group of genes correlated with progression of NAFLD Scarb2, Cd36, Dab2 Gstm5 (up-regulated) and Fmo5 (down-regulated) were included in the group of genes expressed exclusively in NASH.

Acp5, Tspo, Ptgis, Tnfrsf and Gsta5 expression increased among genes correlated with the progression of NAFLD. Acp5 is also known as TRACP $5 \mathrm{a}$, which is the novel marker of macrophage activation and inflammatory disease processes [21,22]. TRACP mRNA and a monomeric TRACP protein, equivalent to serum TRACP 5a, demonstrated more abundant expression in adiposederived macrophages of obese adults in comparison with those of lean individuals [23]. Therefore, elevated Acrp5 may be representative of macrophage infiltration in the liver of SS and NASH model rats.

Tspo is involved in the regulation of cholesterol transport into mitochondria in relation to bile production and steroidogenesis as well as in oxidative stress, apoptosis and inflammatory and immune responses [24-32]. Interestingly, Kugler et al. found that Tspo appears to be an active participant in the generation of Reactive oxygen Species (ROS) at mitochondrial levels and in the modulation of the mitochondrial membrane potential, thereby playing a role in the induction of the mitochondrial apoptosis cascade [33,34]. Increased Tspo may be a key factor in oxidative stress in the liver of SS and NASH model rats. The 4-1BB (also known as ILA; 4-1BB; CD137; CDw137) receptor, a recently identified molecule of the tumor necrosis factor-receptor (Tnfr) superfamily, is a type I membrane protein expressed on activated cytolytic and helper $\mathrm{T}$ cells [35]. The 4-1BB receptor ligand (4-1BBL) is expressed on APCs including B cells, macrophages and dedritic cells $[36,37]$. Ligation of $4-1 \mathrm{BB}$ with $4-1 \mathrm{BBL}$ plays an important role in sustaining $\mathrm{T}$ cell activation and amplifying Cytotoxic T Lymphocyte (CTL) response [38]. Enhanced Tnfr may be related to cytokine signal in the liver of SS and NASH model rats. These data suggested that Acrp5, Tspo and Tnfr were already elevated in liver at the SS stage, which may initiate macrophage infiltration and oxidative stress in the liver. Prostacyclin (PGI2) inhibits platelet aggregation and vasoconstriction. PGI2 synthase (Ptgis), which is widely distributed, occurs predominantly in vascular endothelial and smooth muscle cells [39]. Ptgis is over-expressed and exhibits a strong protective effect against cytokine toxicity, which is correlated with decreased activation of the transcription factor NFkB and the inducible NO synthase promoter as well as reduced inducible NO synthase protein expression and nitrite production. Reduction in the cytokinestimulated endoplasmic reticulum and mitochondrial stress was also observed in the Ptgis-over-expressing cells. As a result, increased Ptgis may function as a protection system in the liver of SS and NASH model 
Citation: Ohba K, Kumai T, Iwai S, Watanabe M, Koizumi G, et al. (2012) Hepatic Gene Expression Associated With Macrophage and Oxidative Stress of Simple Steatosis and Non-Alcoholic Steatohepatitis Model Rats Using DNA Microarray Analysis. J Metabolic Synd 1:115. doi:10.4172/2167-0943.1000115

Page 5 of 7

rats. The Glutathione-S-transferases (GST) comprise a major group of detoxification enzymes, which are ubiquitous in all eukaryotic species [40]. GST is responsible for catalyzing the conjugation of Glutathione (GSH) to a wide spectrum of electrophilic compounds including endogenous substances and xenobiotics [41]. Increased expression of GST in response to exposure to xenobiotics is thought to constitute an adaptive response by the exposed cells to chemical or oxidative stress [42]. Gsta5 may also play a role in the protection system in the NAFLD model liver. It is possible that Ptgis and Gsta5 are up-regulated in compensatory fashion in NAFLD liver as a protective mechanism. Rab8, Rgn and Gstk1 decreased among genes correlated with progression of NAFLD.

Rab8, which regulates endosomal cholesterol removal to apoA-I in human fibroblasts, is abundantly expressed in human atherosclerotic lesion macrophages and up-regulated on lipid loading of macrophages in vitro; additionally, Rab8 reduces foam cell formation by facilitating ABCA1 surface expression and stimulating endosomal cholesterol efflux to apoA-I in primary human macrophages [43]. Rab8 redistributes cholesterol from late endosomes to the cell periphery and stimulates cholesterol efflux to the ABCA1-ligand apolipoprotein A-I (apoA-I) without increasing cholesterol esterification. Depletion of Rab8 from wild-type fibroblasts results in cholesterol deposition within late endosomal compartments [44]. Diminished Rab8 expression may be related to lipid deposition in the liver of SS and NASH model rats.

Rgn (also known as Senescence Marker Protein-30 (SMP30)) acts as an antioxidant and anti-apoptotic protein. Hepatic SMP30 is closely associated with the pathogenesis of NAFLD [45]; over-expression of SMP30/GNL in Hep G2 cells contributes to decreased ROS formation accompanied by declines in lipid peroxidation, SOD activity and GSH levels [46]. SMP30, which is a potential biomarker for the diagnosis and prognosis of acute liver failure, also plays a very important role in a self-protective mechanism in survival and participates in the pathophysiological processes of acute liver failure [47]. Therefore, the down-regulation of Rgn may be representative of increased oxidative stress in the liver of SS and NASH model rats.

The kappa class GST, which occurs specifically within mitochondria and peroxisomes [48], conjugates glutathione to the classic xenobiotic substrate 1-chloro-2,4-dinitrobenzene. Detection of mGstk1 in the mitochondria of hepatic and renal tissue suggests that this kappa class transferase likely functions as an antioxidant in this organelle [49]. These data suggested that the anti-lipid deposition and antioxidant systems are diminished in the liver of SS and NASH model rats. Scarb2, Cd36, Dab2 and Gstm5 increased among genes expressed exclusively in NASH. Scarb2 is also known as AMRF, EPM4, LGP85, CD36L2, HLGP85, LIMP-2, LIMPII and Scavenger receptors type II (SR-BII). Ishikawa et al. [50] previously reported that SR-B type I and II are expressed in macrophages. Foam cell formation of macrophages is mediated by the uncontrolled uptake of modified and oxidized LowDensity Lipoprotein (LDL) via scavenger receptors, which produces excessive lipoprotein-derived Cholesteryl Ester (CE) accumulation [51]. Elevated scarb2 may be indicative of macrophage infiltration in the liver of NASH model animals.

Cd36 was described as a transporter governing the rate-limiting steps of fatty acid uptake on the plasma membrane of hepatocytes [52]. Involvement of the $\mathrm{Cd} 36$ antigen has been demonstrated in phagocytosis of apoptotic cells [53] as well as in the endocytosis of longchain fatty acids, anionic phospholipids and oxidized lipoproteins [54]; moreover, $\mathrm{Cd} 36$ is a class B scavenger receptor. High LDL levels have been shown to become atherogenic when oxidized to modify LDL (OxLDL) by inducing foam cell formation via enhanced Cd36 expression on macrophages. In addition to Ox-LDL, elevated levels of glucose, insulin resistance, low HDL cholesterol and increased levels of free fatty acid (FFA) all result in enhanced expression of CD36, thereby contributing to type 2 diabetes mellitus (T2DM) and related atherosclerosis. Therefore, up-regulation of $\mathrm{Cd} 36$ may lead to progression of lipid deposition in the liver of SS and NASH model rats. The cytokine TGF-beta acts as a tumor suppressor in normal epithelial cells during the early stages of tumorigenesis. It was suggested that down-regulation of Dab2 blocks TGF-beta-mediated inhibition of cell proliferation and migration and enables TGF-beta promotion of cell motility, anchorage-independent growth and tumor growth in vivo [55]. Up-regulation of Dab2 may be related to cytokine signaling in the liver of SS and NASH model rats.

Members of the GST isoenzyme families, alpha, mu and pi, are elevated in response to chemical and oxidative stress [56]. An increasing volume of data suggests that African-Americans with NAFLD tend to display less progressive liver disease. In comparison to Caucasian NASH patients, African-American NASH patients exhibit over-expression of GSTM 2, GSTM4, GSTM5, FH and ASCL4 [57]. Over-expression of glutathione S-transferase mu transcripts (GSTM1, GSTM3, GSTM4 and GSTM5) may contribute to a decrease in oxidative stress [58]. These findings suggested that increased Scarb2, Cd36 and Dab2 may be related to pathogenesis of NASH and that GSTm5 may be enhanced in compensatory fashion.

Fmo5, which belongs to a family of enzymes that catalyzes the oxygenation of nucleophilic $\mathrm{N}$ - and S-containing compounds, decreased among genes expressed exclusively in NASH. The FMO enzyme family consists of five forms (FMOs1-5) that share approximately 50-60\% sequence identity with one another [59]. Fmo1, Fmo3 and Fmo5 mRNAs were also found to be down-regulated in LPS models of inflammation [60]. Toll-Like Receptor (TLR) 4 is responsible for LPS signaling in association with several proteins. The down-regulation of Fmo3 and Fmo5 in this model is TLR4-dependent [61]. These data suggested that decreased Fmo5 may be related to inflammation of NASH liver.

\section{Conclusion}

Hepatic gene expressions in NAFLD and NASH model rats were elucidated. We hypothesized that scavenger receptor class $\mathrm{B}$ and Glutathione S-transferase (GST) play important roles in the progression from simple NAFLD to NASH. The current data afford beneficial information regarding therapeutic targets of NAFLD/NASH progression. Additional studies are necessary in order to confirm the role of these genes.

\section{Acknowledgement}

We are grateful for the support from the Showa University Research Grant for Young Researchers.

\section{References}

1. McCullough AJ (2004) The clinical features, diagnosis and natural history of nonalcoholic fatty liver disease. Clin Liver Dis 8: 521-533.

2. Dowman JK, Tomlinson JW, Newsome PN (2011) Systematic review: the diagnosis and staging of non-alcoholic fatty liver disease and non-alcoholic steatohepatitis. Aliment Pharmacol Ther 33: 525-540.

3. Brunt EM, Janney CG, Di Bisceglie AM, Neuschwander-Tetri BA, Bacon BR (1999) Nonalcoholic steatohepatitis: a proposal for grading and staging the histological lesions. Am J Gastroenterol 94: 2467-2474.

4. Edmison JM, Kalhan SC, McCullough AJ (2009) Obesity, hepatic metabolism and disease. Nestle Nutr Workshop Ser Pediatr Program 63: 163-172.

5. Siegel AB, Zhu AX (2009) Metabolic syndrome and hepatocellular carcinoma: two growing epidemics with a potential link. Cancer 115: 5651-5661. 
Citation: Ohba K, Kumai T, Iwai S, Watanabe M, Koizumi G, et al. (2012) Hepatic Gene Expression Associated With Macrophage and Oxidative Stress of Simple Steatosis and Non-Alcoholic Steatohepatitis Model Rats Using DNA Microarray Analysis. J Metabolic Synd 1:115. doi:10.4172/2167-0943.1000115

Page 6 of 7

6. Adams LA, Lymp JF, St Sauver J, Sanderson SO, Lindor KD, et al. (2005) The natural history of nonalcoholic fatty liver disease: a population-based cohort study. Gastroenterology 129: 113-121.

7. Petta S, Muratore C, Craxi A (2009) Non-alcoholic fatty liver disease pathogenesis: the present and the future. Dig Liver Dis 41: 615-625

8. Dowman JK, Tomlinson JW, Newsome PN (2010) Pathogenesis of nonalcoholic fatty liver disease. QJM 103: 71-83

9. Kumai T, Oonuma S, Kitaoka Y, Tadokoro M, Kobayashi S (2003) Biochemical and morphological characterization of spontaneously hypertensive hyperlipidaemic rats. Clin Exp Pharmacol Physiol 30: 537-544.

10. Kumai T, Oonuma S, Matsumoto N, Takeba Y, Taniguchi R, et al. (2004) Antilipid deposition effect of HMG-CoA reductase inhibitor, pitavastatin, in a rat model of hypertension and hypercholesterolemia. Life Sci 74: 2129-2142.

11. Saiki R, Okazaki M, Iwai S, Kumai T, Kobayashi S, et al. (2007) Effects of pioglitazone on increases in visceral fat accumulation and oxidative stress in spontaneously hypertensive hyperlipidemic rats fed a high-fat diet and sucrose solution. J Pharmacol Sci 105: 157-167.

12. Tomita Y, Iwai S, Kumai T, Ohnuma S, Kurahashi C, et al. (2010) Viscera fat accumulation is associated with oxidative stress and increased matrix metalloproteinase- 9 expression in atherogenic factor-overlapped model rats. Showa Univ J Med Sci 22: 27-40.

13. Tsuchiya H, Iwai S, Kumai T, Ohnuma S, Tsuboi A, et al. (2011) Increase in matrix metalloproteinase-2 and 9 in the liver of nonalcoholic steatohepatitis (NASH) model rats. Showa Univ J Med Sci 23: 37-50.

14. Matsuzawa Y (1997) Pathophysiology and molecular mechanisms of viscera fat syndrome: the Japanese experience. Diabetes Metab Rev 13: 3-13.

15. Kurukulasuriya LR, Govindarajan G, Sowers J (2006) Stroke prevention in diabetes and obesity. Expert Rev Cardiovasc Ther 4: 487-502

16. Eguchi Y, Mizuta T, Sumida Y, Ishibashi E, Kitajima Y, et al. (2011) The pathological role of visceral fat accumulation in steatosis, inflammation, and progression of nonalcoholic fatty liver disease. J Gastroenterol 46: 70-78.

17. Sakaguchi S, Takahashi S, Sasaki T, Kumagai T, Nagata K (2011) Progression of alcoholic and non-alcoholic steatohepatitis: common metabolic aspects of innate immune system and oxidative stress. Drug Metab Pharmacokinet 26 $30-46$

18. Day CP, James OF (1998) Steatohepatitis: a tale of two "hits"? Gastroenterology 114: 842-845.

19. Bieghs V, Rensen PC, Hofker MH, Shiri-Sverdlov R (2012) NASH and atherosclerosis are two aspects of a shared disease: central role for macrophages. Atherosclerosis 220: 287-293.

20. Koek GH, Liedorp PR, Bast A (2011) The role of oxidative stress in nonalcoholic steatohepatitis. Clin Chim Acta 412: 1297-1305.

21. Shidara K, Inaba M, Okuno S, Yamada S, Kumeda Y, et al. (2008) Serum levels of TRAP5b, a new bone resorption marker unaffected by renal dysfunction, as a useful marker of cortical bone loss in hemodialysis patients. Calcif Tissue Int 82: $278-287$

22. Janckila AJ, Slone SP, Lear SC, Martin A, Yam LT (2007) Tartrate-resistan acid phosphatase as an immunohistochemical marker for inflammatory macrophages. Am J Clin Pathol 127: 556-566.

23. Lang P, van Harmelen V, Ryden M, Kaaman M, Parini P, et al. (2008) Monomeric tartrate resistant acid phosphatase induces insulin sensitive obesity. PLoS One 3: e1713.

24. Gavish M, Bar-Ami S, Weizman R (1992) The endocrine system and mitochondrial benzodiazepine receptors. Mol Cell Endocrinol 88: 1-13.

25. Knudsen J, Mandrup S, Rasmussen JT, Andreasen PH, Poulsen F, et al. (1993) The function of acyl-CoA-binding protein (ACBP)/diazepam binding inhibito (DBI). Mol Cell Biochem 123: 129-138.

26. Krueger KE, Papadopoulos V (1990) Peripheral-type benzodiazepine receptors mediate translocation of cholesterol from outer to inner mitochondrial membranes in adrenocortical cells. J Biol Chem 265: 15015-15022.

27. Kunduzova OR, Escourrou G, De La Farge F, Salvayre R, Seguelas MH, et al. (2004) Involvement of peripheral benzodiazepine receptor in the oxidative stress, death-signaling pathways, and renal injury induced by ischemiareperfusion. J Am Soc Nephrol 15: 2152-2160.
28. Papadopoulos V, Baraldi M, Guilarte TR, Knudsen TB, Lacapere JJ, et al. (2006) Translocator protein (18kDa): new nomenclature for the peripheral-type benzodiazepine receptor based on its structure and molecular function. Trends Pharmacol Sci 27: 402-409.

29. Veenman L, Gavish M (2006) The peripheral-type benzodiazepine receptor and the cardiovascular system. Implications for drug development. Pharmacol The 110: 503-524

30. Veenman L, Papadopoulos V, Gavish M (2007) Channel-like functions of the 18$\mathrm{kDa}$ translocator protein (TSPO): regulation of apoptosis and steroidogenesis as part of the host-defense response. Curr Pharm Des 13: 2385-2405.

31. Veenman L, Shandalov Y, Gavish M (2008) VDAC activation by the $18 \mathrm{kDa}$ translocator protein (TSPO), implications for apoptosis. J Bioenerg Biomemb 40: $199-205$

32. Veenman L, Alten J, Linnemannstons $\mathrm{K}$, Shandalov $\mathrm{Y}$, Zeno S, et al. (2010) Potential involvement of FOF1-ATP(synth)ase and reactive oxygen species in apoptosis induction by the antineoplastic agent erucylphosphohomocholine in glioblastoma cell lines : a mechanism for induction of apoptosis via the $18 \mathrm{kDa}$ mitochondrial translocator protein. Apoptosis 15: 753-768.

33. Kugler W, Veenman L, Shandalov Y, Leschiner S, Spanier I, et al. (2008) Ligands of the mitochondrial $18 \mathrm{kDa}$ translocator protein attenuate apoptosis of human glioblastoma cells exposed to erucylphosphohomocholine. Cell Oncol 30: $435-450$

34. Zeno S, Zaaroor M, Leschiner S, Veenman L, Gavish M (2009) CoCl(2) induces apoptosis via the $18 \mathrm{kDa}$ translocator protein in U118MG human glioblastoma cells. Biochemistry 48: 4652-4661.

35. Kwon BS, Weissman SM (1989) cDNA sequences of two inducible T-cell genes. Proc Natl Acad Sci U S A 86: 1963-1967.

36. Pollok KE, Kim YJ, Hurtado J, Zhou Z, Kim KK, et al. (1994) 4-1BB T-cell antigen binds to mature $B$ cells and macrophages, and costimulates anti-muprimed splenic B cells. Eur J Immunol 24: 367-374.

37. DeBenedette MA, Shahinian A, Mak TW, Watts TH (1997) Costimulation of CD28- T lymphocytes by 4-1BB ligand. J Immunol 158: 551-559.

38. Chu NR, DeBenedette MA, Stiernholm BJ, Barber BH, Watts TH (1997) Role of IL-12 and 4-1BB ligand in cytokine production by CD28+ and CD28- T cells J Immunol 158: 3081-3089.

39. Nakayama T (2010) Genetic polymorphisms of prostacyclin synthase gene and cardiovascular disease. Int Angiol 29: 33-42

40. Kazi S, Ellis EM (2002) Expression of rat liver glutathione-S-transferase GSTA5 in cell lines provides increased resistance to alkylating agents and toxic aldehydes. Chem Biol Interact 140: 121-135.

41. Booth J, Boyland E, Sims P (1961) An enzyme from rat liver catalysing conjugations with glutathione. Biochem J 79: 516-524.

42. Hayes JD, Ellis EM, Neal GE, Harrison DJ, Manson MM (1999) Cellula response to cancer chemopreventive agents: contribution of the antioxidant responsive element to the adaptive response to oxidative and chemical stress. Biochem Soc Symp 64: 141-168.

43. Linder MD, Mayranpaa MI, Peranen J, Pietila TE, Pietiainen VM, et al. (2009) Rab8 regulates ABCA1 cell surface expression and facilitates cholesterol efflux in primary human macrophages. Arterioscler Thromb Vasc Biol 29: 883-888.

44. Linder MD, Uronen RL, Holtta-Vuori M, van der Sluijs P, Peranen J, et al. (2007) Rab8-dependent recycling promotes endosomal cholesterol removal in normal and sphingolipidosis cells. Mol Biol Cell 18: 47-56.

45. Park H, Ishigami A, Shima T, Mizuno M, Maruyama N, et al. (2010) Hepatic senescence marker protein-30 is involved in the progression of nonalcoholic fatty liver disease. J Gastroenterol 45: 426-434.

46. Handa S, Maruyama N, Ishigami A (2009) Over-expression of Senescence Marker Protein-30 decreases reactive oxygen species in human hepatic carcinoma Hep G2 cells. Biol Pharm Bull 32: 1645-1648.

47. Lv S, Wang JH, Liu F, Gao Y, Fei R, et al. (2008) Senescence marker protein 30 in acute liver failure: validation of a mass spectrometry proteomics assay. BMC Gastroenterol 8: 17.

48. Morel F, Rauch C, Petit E, Piton A, Theret N, et al. (2004) Gene and protein characterization of the human glutathione S-transferase kappa and evidence for a peroxisomal localization. J Biol Chem 279: 16246-16253.

49. Thomson RE, Bigley AL, Foster JR, Jowsey IR, Elcombe CR, et al. (2004) 
Citation: Ohba K, Kumai T, Iwai S, Watanabe M, Koizumi G, et al. (2012) Hepatic Gene Expression Associated With Macrophage and Oxidative Stress of Simple Steatosis and Non-Alcoholic Steatohepatitis Model Rats Using DNA Microarray Analysis. J Metabolic Synd 1:115. doi:10.4172/2167-0943.1000115

Page 7 of 7

Tissue-specific expression and subcellular distribution of murine glutathione S-transferase class kappa. J Histochem Cytochem 52: 653-662.

50. Ishikawa Y, Kimura-Matsumoto M, Murakami M, Yamamoto K, Akasaka Y, et al. (2009) Distribution of smooth muscle cells and macrophages expressing scavenger receptor $\mathrm{BI} / \mathrm{II}$ in atherosclerosis. J Atheroscler Thromb 16: 829-839.

51. de Villiers WJ, Smart EJ (1999) Macrophage scavenger receptors and foam cell formation. J Leukoc Biol 66: 740-746.

52. Stahlberg N, Rico-Bautista E, Fisher RM, Wu X, Cheung L, et al. (2004) Femalepredominant expression of fatty acid translocase/CD36 in rat and human liver. Endocrinology 145: 1972-1979.

53. Ren Y, Silverstein RL, Allen J, Savill J (1995) CD36 gene transfer confers capacity for phagocytosis of cells undergoing apoptosis. J Exp Med 181: 18571862.

54. Rigotti A, Acton SL, Krieger M (1995) The class B scavenger receptors SR-B and CD36 are receptors for anionic phospholipids. J Biol Chem 270: 16221 16224

55. Hannigan A, Smith P, Kalna G, Lo Nigro C, Orange C, et al. (2010) Epigenetic downregulation of human disabled homolog 2 switches TGF-beta from a tumor suppressor to a tumor promoter. J Clin Invest 120: 2842-2857.
56. Hayes JD, Wolf CR (1990) Molecular mechanisms of drug resistance. Biochem J 272: 281-295.

57. Stepanova M, Hossain N, Afendy A, Perry K, Goodman ZD, et al. (2010) Hepatic gene expression of Caucasian and African-American patients with obesity-related non-alcoholic fatty liver disease. Obes Surg 20: 640650

58. Raza A, Dikdan G, Desai KK, Shareef A, Fernandes H, et al. (2010) Globa gene expression profiles of ischemic preconditioning in deceased donor liver transplantation. Liver Transpl 16: 588-599.

59. Motika MS, Zhang J, Ralph EC, Dwyer MA, Cashman JR (2012) pH dependence on functional activity of human and mouse flavin-containing monooxygenase 5 . Biochem Pharmacol 83: 962-968.

60. Zhang J, Chaluvadi MR, Reddy R, Motika MS, Richardson TA, et al. (2009) Hepatic flavin-containing monooxygenase gene regulation in different mouse inflammation models. Drug Metab Dispos 37: 462-468.

61. Koutoulaki A, Langley M, Sloan AJ, Aeschlimann D, Wei XQ (2010) TNFalpha and TGF-beta1 influence IL-18-induced IFNgamma production through regulation of IL-18 receptor and T-bet expression. Cytokine 49: 177-184. 